\title{
Enhanced Universal Dependency Parsing with Second-Order Inference and Mixture of Training Data
}

\author{
Xinyu Wang ${ }^{\diamond}$, Yong Jiang ${ }^{\dagger}$, Kewei Tu ${ }^{\diamond}$ \\ ${ }^{\diamond}$ School of Information Science and Technology, ShanghaiTech University \\ Shanghai Engineering Research Center of Intelligent Vision and Imaging \\ ${ }^{\dagger}$ DAMO Academy, Alibaba Group \\ \{wangxy 1, tukw @shanghaitech.edu.cn \\ yongjiang.jy@alibaba-inc.com
}

\begin{abstract}
This paper presents the system used in our submission to the IWPT 2020 Shared Task. Our system is a graph-based parser with secondorder inference. For the low-resource Tamil corpus, we specially mixed the training data of Tamil with other languages and significantly improved the performance of Tamil. Due to our misunderstanding of the submission requirements, we submitted graphs that are not connected, which makes our system only rank 6th over 10 teams. However, after we fixed this problem, our system is 0.6 ELAS higher than the team that ranked 1st in the official results.
\end{abstract}

\section{Introduction}

Based on the Universal Dependencies (UD) (Nivre et al., 2016), the Enhanced Universal Dependencies (EUD) (Bouma et al., 2020) ${ }^{1}$ are non-tree graphs with reentrancies, cycles, and empty nodes to deal with the problem that purely rooted trees cannot adequately represent grammatical relations. We found that we can reduce parsing such a graph to parsing bi-lexical structures like semantic dependency parsing (SDP) (Oepen et al., 2015) by reducing reentrancies and empty nodes into new labels. (Wang et al., 2019) is a state-of-the-art approach for the semantic dependency parsing tasks that use second-order inference methods with Mean-Field Variational Inference. We adopt their approach for decoding and encode the sentences with strong pretrained token representations: XLMR (Conneau et al., 2019), Flair (Akbik et al., 2018) and FastText (Bojanowski et al., 2017). Among the datasets, the Tamil language only contains 400 labeled sentences for training, which makes the performance of the model for Tamil low. To further improve

\footnotetext{
${ }^{1}$ https://universaldependencies.org/u/ overview/enhanced-syntax.html
}

the performance for the low resource language, we propose a new approach that we train the Tamil model with a mixture of datasets with Tamil and a rich resource language. Empirical results show that such an approach can improve 2.44 ELAS on the test set of Tamil. Due to our misconceptions on the submission format, we submitted invalid unconnected graphs to the submission site. Thanks to the help of the organizers, they fixed these graphs with simple scripts, and our system is ranked 6th over 10 teams in the official results. However, the submitted graphs can be easily connected if we apply tree algorithms in the decoding. In the postevaluation, we submitted our system outputs again and found that our system is 0.56 ELAS higher than the team ranked $\mathbf{1 s t}$ in the official results.

\section{System Description}

\subsection{Data Pre-processing}

There are two features in the EUD graphs that do not appear in SDP graphs. One is the reentrancies of the same head and dependent on different labels. We combined these arcs into one and concatenate the labels of these arcs with a symbol ' + ' representing the combination of two arcs. In the postprocessing, we split arcs with the " + " symbol in the corresponding labels into multiple arcs. Another one is the empty nodes that are introduced in the shared task (for example, nodes with id 1.1). We used the official script to collapse graphs through reducing such empty nodes into non-empty nodes and introducing new dependency labels ${ }^{2}$. In the post-process, we add empty nodes according to the dependency labels. As the official evaluation only score the collapsed graphs, such a process does not impact the system performance.

\footnotetext{
${ }^{2}$ For more details, please refer to https: //universaldependencies.org/iwpt20/task_ and_evaluation.html.
} 


\subsection{Approach}

We follow the approach of Wang et al. (2019) ${ }^{3}$ to build our system which uses the second-order inference algorithm for the arc predictions. Given a sentence with $n$ words $\mathbf{w}=\left[w_{1}, w_{2}, \ldots, w_{n}\right]$, we feed a three-layer BiLSTM with their corresponding token representations.

$$
\mathbf{R}=\operatorname{BiLSTM}(\mathbf{E})
$$

where $\mathbf{E}=\left[\mathbf{e}_{1}, \ldots, \mathbf{e}_{n}\right]$ is the concatenation of various embeddings of token (We use different combination of XLMR, Flair and FastText for each language as the token representation.) and $\mathbf{R}=\left[\mathbf{r}_{1}, \ldots, \mathbf{r}_{n}\right]$ represents the output from the BiLSTM. For the arc predictions, we use the feedforward network, Biaffine and Trilinear functions to encode unary potentials $\psi_{u}$ and binary potentials $\psi_{b}$ :

$$
\begin{aligned}
\psi_{u}\left(w_{i}, w_{j}\right) & =\text { FNN_Biaffine } \\
\psi_{b}\left(w_{i}, w_{j}, w_{k}\right) & =\text { FNN }\left(\mathbf{r}_{i}, \mathbf{r}_{j}\right) \\
&
\end{aligned}
$$

where FNN_Biaffine and FNN_Trilinear represent a combination of FNN and Biaffine/Trilinear functions. Then we feed these potentials into a Mean-Field Variational Inference network for the second-order inference.

$$
P(\mathbf{Y} \mid \mathbf{w})=\operatorname{MFVI}\left(\psi_{u}, \psi_{p}\right)
$$

where $P(\mathbf{Y} \mid \mathbf{w})$ is a probability matrix representing the probabilities of all potential arcs. We first use tree algorithms like the Eisner's (Eisner, 2000) or MST (McDonald et al., 2005) algorithms to ensure the connectivity of the graph. Then we additionally add arcs for the positions that $P(\mathbf{Y} \mid \mathbf{w})>0.5$. For the label predictions, we use the FNN_Biaffine to score the labels for each potential arc.

$$
\begin{gathered}
\mathbf{s}_{i j}^{\text {(label) }}=\text { FNN_Biaffine } \\
{ }^{(\text {label })}\left(\mathbf{r}_{i}, \mathbf{r}_{j}\right) \\
P^{(\text {label })}\left(y_{i j} \mid \mathbf{w}\right)=\operatorname{softmax}\left(\mathbf{s}_{i j}^{(\text {label })}\right)
\end{gathered}
$$

We select the label with the highest score of each potential arc.

To train the system, we follow the approach of Wang et al. (2019) with the cross entropy loss:

$$
\begin{aligned}
\mathcal{L}^{(\text {arc })}(\theta) & =-\sum_{i, j} \log \left(P_{\theta}\left(y_{i j}^{\star(\operatorname{arc})} \mid \mathbf{w}\right)\right) \\
\mathcal{L}^{(\text {label })}(\theta) & =-\sum_{i, j} \mathbb{1}\left(y_{i j}^{\star(\text { arc })}\right) \log \left(P_{\theta}\left(y_{i j}^{\star(\text { label })} \mid \mathbf{w}\right)\right)
\end{aligned}
$$

\footnotetext{
${ }^{3}$ https://github.com/wangxinyu0922/ Second_Order_SDP
}

where $\theta$ is the parameters of our system, $\mathbb{1}\left(y_{i j}^{\star(\operatorname{arc})}\right)$ denotes the indicator function and equals 1 when edge $(i, j)$ exists in the gold parse and 0 otherwise, and $i, j$ ranges over all the tokens $\mathbf{w}$ in the sentence. The two losses are combined by a weighted average.

$$
\mathcal{L}=\lambda \mathcal{L}^{(\text {label })}+(1-\lambda) \mathcal{L}^{(a r c)}
$$

\subsection{Mixture of Datasets for Tamil Parser Training}

Tamil dataset has the fewest training and development sentences over all languages, which contains 400 sentences for training and 80 sentences for development. Therefore we believe that Tamil parser can be easily improved if we use more training data. With the emergence of multilingual contextual embeddings like multilingual BERT (Devlin et al., 2019) and XLMR, training a unified multilingual model with high performances over all languages becomes possible through mixing the training data of multiple languages. However, it does not apply to the shared task as the label set of EUD is distinct in different languages. The arc annotations in the dataset are still helpful for training the Tamil parser. Thus we removed the label annotations in the dataset of other languages so that the label loss of these data cannot be back-propagated. Then we mixed one of the languages with the fully annotated Tamil dataset. To solve the problem of data imbalance in the mixture of the dataset in training, we upsampled the Tamil training set to keep the same data size as that of the other language.

\section{Settings and Results}

\subsection{Experimental Settings}

In training, we split the official development set into halves as the development set and test set. We used the development set to select the model based on labeled F1 score which is the metric used in the SDP task and it evaluates the accuracy of predicted labeled arcs. We used the test set to choose the best model architecture. We use a batch size of 2000 tokens with the Adam (Kingma and Ba, 2015) optimizer. The hyper-parameters of our system are shown in Table 1, which are mostly adopted from previous work on dependency parsing. We only use the tokenized words as the model input. For the Tamil Parser, we tried English or Czech datasets to mix with the Tamil dataset. For most of languages, we used freezed XLMR embedding 


\begin{tabular}{lr}
\hline \hline Hidden Layer & Hidden Sizes \\
\hline BiLSTM LSTM & $3 * 400$ \\
Unary Arc/Label & 500 \\
Binary Arc & 150 \\
Embedding/LSTM Dropouts & $33 \%$ \\
Loss Interpolation $(\lambda)$ & 0.10 \\
Adam $\beta_{1}$ & 0.9 \\
Adam $\beta_{2}$ & 0.9 \\
Learning rate & $2 e^{-3}$ \\
LR decay & 0.5 \\
\hline \hline
\end{tabular}

Table 1: Hyper-parameters for our system.

only as we found that the Flair embeddings and FastText embeddings were not helpful for the task except Tamil. We used a concatenate of XLMR, Flair and FastText embeddings for Tamil parser training. For the sentence and word segmentation, we used Stanza (Qi et al., 2020) models that were trained on treebank with the largest training set for all languages except Lithuanian, because the model trained on the Lithuanian-HSE treebank has an extremely low segmentation performance compared with the model trained on Lithuanian-ALKSNIS.

\subsection{Main Results}

Table 2 shows the results of official evaluations of all teams, as well as the post-evaluations of our system. In the Official submission, we trained the Tamil Parser with a mixture of English and Tamil datasets ('Ours+en+MST' in the table), and in the post-evaluation, we also tried a mixture of Czech and Tamil datasets ('Ours+cs+MST' in the table) because the Czech dataset contains the largest training data over all languages. In the official results, our system was fixed by the organizers through their simple scripts for the connectivity of graphs, which significantly reduced our system performance. In the post-evaluation, we fixed this issue with MST or Eisner's algorithm and showed that our system performs 0.6 ELAS higher than the best team. For the Tamil parser, mixing the Tamil dataset with the Czech dataset performs 1.7 ELAS better than mixing with the English dataset, which shows that a larger dataset gives better results than the smaller one. Our system with the MST algorithm is 0.2 ELAS stronger than the system with Eisner's algorithm, which shows that the non-projective tree algorithm (MST) is better than the projective tree algorithm (Eisner's) for the EUD task. We built our codes based on PyTorch (Paszke et al., 2019), and ran our experiments on a single Tesla V100 GPU.

\subsection{Comparison of First-Order and Second-Order Inference}

Table 3 shows a performance comparison between two kinds of embedding choices, XLMR+Flair+FastText and XLMR, and first-order and second-order inference. The results show that second-order inference is stronger than first-order inference in all languages, and embeddings with XLMR embedding only usually perform better than XLMR+Flair+FastText embeddings. However, the Flair+FastText embedding is helpful for Tamil. Therefore we use XLMR+Flair+FastText embeddings for training the Tamil parser while we use XLMR embedding only for other languages.

\subsection{Performance Comparison between Connected Graphs and Non-Connected Graphs}

Before the deadline of the shared task, the submission site showed the scores of each treebank separately even the submission graphs were not connected, which unfortunately made us believe that the non-connected graphs are also acceptable for the task. In fact, these graphs are not acceptable and the organizers fixed the issue with some simple scripts, and this results in a significant reduction in the final scores. In section 3.2, we show that appending a tree-parsing algorithm to our system produces connected graphs with high scores. Here we also evaluate the non-connected graphs produced by our original system. We think evaluating non-connected graphs is informative for two reasons. The first is that these results help to understand how different the connected graphs and non-connected graphs performs. The second is that in practice, non-connected graphs can be predicted with a relatively faster speed as the MST and Eisner's algorithms are slow while we can get the non-connected graphs through argmax operations. We compare the performance of connected and non-connected graphs for each treebank and each language in Table 4 and 5. The results show that the non-connected graphs perform slightly better than graphs with the tree algorithms. Therefore generating non-connected trees are more practical in practice if there are no such constraints. 


\begin{tabular}{|c|c|c|c|c|c|c|c|c|c|c|c|c|c|c|c|c|c|c|}
\hline Team Name & ar & $\overline{b g}$ & cs & $\overline{\mathrm{nl}}$ & en & et & $\overline{\text { fi }}$ & $\overline{\mathrm{fr}}$ & it & lv & lt & $\overline{\mathrm{pl}}$ & $\mathrm{ru}$ & $\overline{\mathrm{sl}}$ & $\overline{\text { sv }}$ & ta & $\overline{\mathrm{uk}}$ & Avg. \\
\hline & \multicolumn{18}{|c|}{ Official } \\
\hline Rob & & 0.0 & 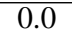 & 0 & .9 & 0 & 0.0 & 0.0 & 0.0 & 0.0 & 0.0 & 0.0 & .0 & 0.0 & .0 & 0 & 0.0 & 5.2 \\
\hline Koel & & 68.9 & 61.1 & 62.9 & 5.4 & 59.1 & 7.5 & 67.9 & 69.1 & 64.8 & 56.3 & 61.3 & 64.2 & 64.1 & 4.5 & 47.4 & 64.2 & 62.9 \\
\hline & & 77.3 & 5.4 & 67.7 & 0.4 & 61.1 & 72.4 & 74.7 & 72.0 & 72.4 & 58.4 & 65.9 & 75.3 & 68.4 & 3.4 & 48.5 & 6.4 & 67.2 \\
\hline & & 84.9 & .1 & 78.9 & 2.9 & 60.4 & 66.0 & 72.8 & 87.1 & 66.0 & 52.6 & 71.2 & 70.4 & .2 & .4 & 42 & .2 & 67.9 \\
\hline & & 78.7 & 4 & 70.9 & 2.3 & 74.9 & 0 & 77.0 & 73 & 77.8 & 9 & .0 & 3 & 1 & & 2 & .0 & 71.7 \\
\hline & & 84.9 & 0 & 77.6 & 4.0 & 57.2 & 1 & 78.9 & 89 & 68.2 & 1 & 70.6 & 76.9 & 4 & 7 & 48 & 3.9 & 72.8 \\
\hline & & 84.9 & 2 & 77.4 & 7 & 74 & 75.7 & 77.8 & 84 & 75.6 & 61.4 & 74.5 & 80.4 & 73.5 & 75.2 & 47.0 & 4.0 & 74.0 \\
\hline & 3 & 88.2 & 5.5 & 80.7 & 85.3 & 81.4 & 83.0 & 86.2 & 88.5 & 79.2 & 66.1 & 82.4 & 88.6 & 82.7 & 78.2 & 54.3 & 79.7 & 79.8 \\
\hline & 1.0 & 89.4 & 7.0 & 85.1 & 0 & 81.0 & 86.2 & 83.6 & 90.8 & 82.1 & 75.9 & 80.4 & 89.8 & 84.4 & 83.3 & 64.2 & 84.6 & 82.6 \\
\hline \multirow[t]{2}{*}{ TurkuNLP } & 7.8 & 90.7 & 7.5 & 84.7 & 87.2 & 84.5 & 89.5 & 85.9 & 91.5 & 84.9 & 77.6 & 84.6 & 90.7 & 88.6 & 85.6 & 57.8 & 87.2 & 84.5 \\
\hline & \multicolumn{18}{|c|}{ Post-Evaluation } \\
\hline Oul & 7.7 & 1.5 & 0.1 & 86.2 & 7.1 & 86.0 & 89.0 & 85.3 & 91.5 & 87.6 & 78.9 & 84.0 & 92.3 & 87.6 & 34.7 & 56.7 & 8.0 & 85.0 \\
\hline & 77.8 & 91.1 & 95 & 86 & 87.2 & 85 & 88.5 & 85.3 & 91.5 & 87.3 & 78.6 & 83.7 & 92.3 & 87.1 & 84.8 & 58.4 & 8.0 & 84.9 \\
\hline Ours+cs+MST & 77.7 & 91.5 & 90.1 & 86.2 & 87.1 & 86.0 & 89.0 & 85.3 & 01 & 87.6 & 78.9 & 84.0 & 92.3 & 87.6 & 84.7 & 58.4 & 88.0 & 85.1 \\
\hline
\end{tabular}

Table 2: Official evaluations of all systems and post-evaluations of our team in ELAS. We use the ISO 639-1 language code to represent each language. MST and Eis means the MST and Eisner's algorithm that we used for decoding. 'en' and 'cs' represents which dataset we mixed with the Tamil dataset for training the Tamil parser. Note that 'Ours+en+MST' represent the parsed results of parsers that we used in the Official submission.

\begin{tabular}{l||c|c|c|c|c|c|c|c|c}
\hline \hline Approach & ar & bg & cs & nl & en & et & fi & fr & it \\
\hline \hline XLMR+Flair+FastText+1st-Order & 81.66 & 89.29 & 91.04 & 92.55 & 89.74 & 88.33 & 89.40 & 90.64 & 91.94 \\
XLMR+Flair+FastText+2nd-Order & 81.98 & 89.43 & $\mathbf{9 1 . 3 9}$ & $\mathbf{9 2 . 6 8}$ & 89.58 & $\mathbf{8 8 . 6 9}$ & 89.54 & 91.08 & $\mathbf{9 1 . 9 8}$ \\
XLMR+1st-Order & 82.02 & 90.15 & 90.80 & 92.43 & 90.05 & 88.13 & 89.51 & 91.14 & 91.96 \\
XLMR+2nd-Order & $\mathbf{8 2 . 4 2}$ & $\mathbf{9 0 . 3 7}$ & 91.21 & 92.66 & $\mathbf{9 0 . 2 6}$ & 88.60 & 90.35 & $\mathbf{9 1 . 6 9}$ & $\mathbf{9 1 . 9 8}$ \\
\hline & lv & lt & pl & ru & sk & sv & ta & uk & Avg. \\
\hline \hline XLMR+Flair+FastText+1st-Order & 88.21 & 80.21 & 86.91 & 92.88 & 87.28 & 85.52 & 66.17 & 88.26 & 89.40 \\
XLMR+Flair+FastText+2nd-Order & 88.59 & 81.25 & 86.46 & $\mathbf{9 3 . 2 8}$ & 87.18 & 85.63 & $\mathbf{6 8 . 7 6}$ & 88.04 & 89.59 \\
XLMR+1st-Order & 89.62 & 81.92 & 85.73 & 92.86 & 88.48 & 86.36 & 63.28 & 88.96 & 89.57 \\
XLMR+2nd-Order & $\mathbf{8 9 . 9 7}$ & $\mathbf{8 3 . 2 4}$ & $\mathbf{8 7 . 4 9}$ & 93.21 & $\mathbf{8 9 . 0 7}$ & $\mathbf{8 6 . 8 5}$ & 64.84 & $\mathbf{8 9 . 9 9}$ & $\mathbf{8 9 . 9 5}$ \\
\hline \hline
\end{tabular}

Table 3: A comparison of different word embedding concatenation and first-order and second-order inference approaches on the development set split by ourselves. We report Labeled F1 score (LF1) here.

\begin{tabular}{l||cccccccc}
\hline \hline Graph & ar-PADT & bg-BTB & cs-FicTree & cs-CAC & cs-PDT & cs-PUD & nl-Alpino & nl-LassySmall \\
\hline Non-Connected & 77.74 & $\mathbf{9 1 . 5 0}$ & $\mathbf{9 0 . 6 0}$ & 90.55 & $\mathbf{9 0 . 6 5}$ & $\mathbf{8 4 . 2 6}$ & $\mathbf{9 0 . 1 1}$ & 82.55 \\
MST & 77.73 & 91.48 & 90.51 & $\mathbf{9 0 . 5 9}$ & 90.63 & 84.25 & 90.09 & 82.51 \\
CRF & $\mathbf{7 7 . 7 5}$ & 91.07 & 89.85 & 90.02 & 90.05 & 83.70 & 89.69 & $\mathbf{8 3 . 1 0}$ \\
\hline \hline Graph & en-EWT & en-PUD & et-EDT & et-EWT & fi-TDT & fi-PUD & fr-Sequoia & fr-FQB \\
\hline Non-Connected & 86.33 & $\mathbf{8 8 . 0 5}$ & $\mathbf{8 7 . 3 6}$ & $\mathbf{7 9 . 6 2}$ & $\mathbf{9 0 . 0 0}$ & $\mathbf{8 7 . 5 2}$ & 89.67 & 84.11 \\
MST & 86.30 & $\mathbf{8 8 . 0 5}$ & 87.34 & 79.61 & 89.97 & $\mathbf{8 7 . 5 2}$ & 89.66 & 84.09 \\
CRF & $\mathbf{8 6 . 4 0}$ & 88.04 & 87.07 & 79.42 & 89.44 & 86.97 & $\mathbf{8 9 . 7 3}$ & $\mathbf{8 4 . 1 2}$ \\
\hline \hline Graph & it-ISDT & lv-LVTB & lt-ALKSNIS & pl-LFG & pl-PDB & pl-PUD & ru-SynTagRus & sl-SNK \\
\hline Non-Connected & 91.50 & $\mathbf{8 7 . 6 9}$ & 78.97 & $\mathbf{8 7 . 6 5}$ & $\mathbf{8 3 . 2 3}$ & $\mathbf{8 2 . 9 6}$ & $\mathbf{9 2 . 6 2}$ & $\mathbf{8 7 . 5 6}$ \\
MST & 91.49 & 87.64 & $\mathbf{7 8 . 9 4}$ & $\mathbf{8 7 . 6 5}$ & 83.21 & 82.95 & 92.31 & 87.55 \\
CRF & $\mathbf{9 1 . 5 2}$ & 87.29 & 78.63 & 87.59 & 82.90 & 82.57 & 92.31 & 87.14 \\
\hline \hline Graph & sv-Talbanken & sv-PUD & ta-TTB & uk-IU & Average & & & \\
\hline Non-Connective & 88.35 & $\mathbf{8 0 . 8 8}$ & 56.51 & 88.00 & $\mathbf{8 5 . 5 9}$ & & & \\
MST & 88.33 & 80.87 & 56.56 & $\mathbf{8 8 . 0 2}$ & 85.57 & & & \\
CRF & $\mathbf{8 8 . 3 7}$ & 80.87 & $\mathbf{5 6 . 7 1}$ & $\mathbf{8 8 . 0 2}$ & 85.37 & & & \\
\hline \hline
\end{tabular}

Table 4: A performance comparison in ELAS between non-connected graphs and connected graphs over each treebank on the official test sets.

\subsection{Analysis of Mixture of Training Data}

For a more in-depth comparison of how the combination of different language datasets affects the performance of the Tamil Parser, Table 6 shows that more training data significantly improve the perfor- mance of the parser. We leave for future work other language combinations as well as similar studies of other parsers. 


\begin{tabular}{l||c|c|c|c|c|c|c|c|c}
\hline \hline Team Name & ar & bg & cs & nl & en & et & fi & fr & it \\
\hline TurkuNLP & $\mathbf{7 7 . 8 2}$ & 90.73 & 87.51 & 84.73 & $\mathbf{8 7 . 1 5}$ & 84.54 & $\mathbf{8 9 . 4 9}$ & $\mathbf{8 5 . 9 0}$ & $\mathbf{9 1 . 5 4}$ \\
Ours+en+MST & 77.74 & 91.48 & 90.09 & 86.19 & 87.10 & 85.97 & 88.99 & 85.28 & 91.49 \\
Ours+en & 77.75 & $\mathbf{9 1 . 5 0}$ & $\mathbf{9 0 . 1 1}$ & $\mathbf{8 6 . 2 2}$ & 87.12 & $\mathbf{8 5 . 9 9}$ & 89.01 & 85.29 & 91.50 \\
\hline \hline Team Name & lv & lt & pl & ru & sl & sv & ta & uk & Avg. \\
\hline TurkuNLP & 84.94 & 77.64 & $\mathbf{8 4 . 6 4}$ & 90.69 & $\mathbf{8 8 . 5 6}$ & $\mathbf{8 5 . 6 4}$ & $\mathbf{5 7 . 8 3}$ & 87.22 & 84.50 \\
Ours+en+MST & 87.64 & 78.94 & 84.00 & 92.31 & 87.55 & 84.74 & 56.71 & $\mathbf{8 8 . 0 2}$ & 84.96 \\
Ours+en & $\mathbf{8 7 . 6 9}$ & $\mathbf{7 8 . 9 7}$ & 84.01 & $\mathbf{9 2 . 6 2}$ & 87.56 & 84.75 & 56.52 & 88.00 & $\mathbf{8 4 . 9 8}$ \\
\hline \hline
\end{tabular}

Table 5: A performance comparison in ELAS between non-connected graphs, connected graphs with the MST algorithm and the best system in the official results over each language. Ours+en represents our official submission and evaluated with official evaluation script.

\begin{tabular}{l|cc}
\hline \hline Combination & \# Training Sentences & ELAS \\
\hline Tamil & 400 & 55.39 \\
English+Tamil & $12543+12400$ & 56.56 \\
Czech+Tamil & $102131+102000$ & $\mathbf{5 8 . 4 4}$ \\
\hline \hline
\end{tabular}

Table 6: A comparison between different dataset combinations for the Tamil parser training. The 12000 and 102000 in the \# Training Sentences column represents the upsampled value of 400 labeled sentences in Tamil dataset.

\section{Conclusion}

Our system is a parser with strong contextual embeddings and second-order inference. For the lowresource language, we propose to train the model with a mixture of datasets. Empirical results show that the second-order inference is stronger than the first-order one, and mixing data improves the performance of parser significantly for the lowresource language. After we fix the graph connectivity issue, our system outperforms the system ranked 1st by 0.56 ELAS in the official results. We also show that the non-connected graphs are practically useful for its higher performance and faster speed. Our code is available at https://github. com/Alibaba-NLP/MultilangStructureKD.

\section{References}

Alan Akbik, Duncan Blythe, and Roland Vollgraf. 2018. Contextual string embeddings for sequence labeling. In Proceedings of the 27th International Conference on Computational Linguistics, pages 1638-1649, Santa Fe, New Mexico, USA. Association for Computational Linguistics.

Piotr Bojanowski, Edouard Grave, Armand Joulin, and Tomas Mikolov. 2017. Enriching word vectors with subword information. Transactions of the Association for Computational Linguistics, 5:135-146.

Gosse Bouma, Djamé Seddah, and Daniel Zeman. 2020. Overview of the IWPT 2020 Shared Task on Parsing into Enhanced Universal Dependencies. In Proceedings of the 16th International Conference on Parsing Technologies and the IWPT 2020 Shared Task on Parsing into Enhanced Universal Dependencies, Seattle, US. Association for Computational Linguistics.

Alexis Conneau, Kartikay Khandelwal, Naman Goyal, Vishrav Chaudhary, Guillaume Wenzek, Francisco Guzmán, Edouard Grave, Myle Ott, Luke Zettlemoyer, and Veselin Stoyanov. 2019. Unsupervised cross-lingual representation learning at scale. arXiv preprint arXiv:1911.02116.

Jacob Devlin, Ming-Wei Chang, Kenton Lee, and Kristina Toutanova. 2019. BERT: Pre-training of deep bidirectional transformers for language understanding. In Proceedings of the 2019 Conference of the North American Chapter of the Association for Computational Linguistics: Human Language Technologies, Volume 1 (Long and Short Papers), pages 4171-4186, Minneapolis, Minnesota. Association for Computational Linguistics.

Jason Eisner. 2000. Bilexical grammars and their cubic-time parsing algorithms. In Advances in probabilistic and other parsing technologies, pages 29 61. Springer.

Diederik P Kingma and Jimmy Ba. 2015. Adam: A method for stochastic optimization. In International Conference on Learning Representations.

Ryan McDonald, Koby Crammer, and Fernando Pereira. 2005. Online large-margin training of dependency parsers. In Proceedings of the 43rd Annual Meeting of the Association for Computational Linguistics (ACL'05), pages 91-98.

Joakim Nivre, Marie-Catherine de Marneffe, Filip Ginter, Yoav Goldberg, Jan Hajič, Christopher D. Manning, Ryan McDonald, Slav Petrov, Sampo Pyysalo, Natalia Silveira, Reut Tsarfaty, and Daniel Zeman. 
2016. Universal dependencies v1: A multilingual treebank collection. In Proceedings of the Tenth International Conference on Language Resources and Evaluation (LREC 2016), pages 1659-1666, Portorož, Slovenia. European Language Resources Association (ELRA).

Stephan Oepen, Marco Kuhlmann, Yusuke Miyao, Daniel Zeman, Silvie Cinková, Dan Flickinger, Jan Hajic, and Zdenka Uresova. 2015. Semeval 2015 task 18: Broad-coverage semantic dependency parsing. In Proceedings of the 9th International Workshop on Semantic Evaluation (SemEval 2015), pages 915-926.

Adam Paszke, Sam Gross, Francisco Massa, Adam Lerer, James Bradbury, Gregory Chanan, Trevor Killeen, Zeming Lin, Natalia Gimelshein, Luca Antiga, et al. 2019. Pytorch: An imperative style, high-performance deep learning library. In $A d$ vances in Neural Information Processing Systems, pages $8024-8035$.

Peng Qi, Yuhao Zhang, Yuhui Zhang, Jason Bolton, and Christopher D Manning. 2020. Stanza: A python natural language processing toolkit for many human languages. arXiv preprint arXiv:2003.07082.

Xinyu Wang, Jingxian Huang, and Kewei Tu. 2019. Second-order semantic dependency parsing with end-to-end neural networks. In Proceedings of the 57th Annual Meeting of the Association for Computational Linguistics, pages 4609-4618, Florence, Italy. Association for Computational Linguistics. 